\title{
The "Kiel Knots" Technique for Treatment of Congenital and Acquired Penile Deviation
}

\author{
Daniar K. Osmonov ${ }^{*}$ K. P. Jünemann \\ Department of Urology and Pediatric Urology, University Hospital Schleswig Holstein, Campus Kiel, Kiel, Germany \\ Email: *dosmonov@urology.uni-kiel.de
}

Received November 9, 2012; revised December 11, 2012; accepted December 21, 2012

\begin{abstract}
Objectives: We propose a surgical correction of the penile curvature applying a technique based on the 16-dot plication technique modified by burying the knots in a shallow trough of incised tunica. We entitle this modification the "Kiel Knots”. Material and methods: 20 patients with a penile deviation, average age 36.8 years (24 - 52) were operated. Follow-up time was 26 months. In 8 patients the deviation was congenital, in 12 patients it was an acquired deviation. The deviation was $>30^{\circ}$ in all patients. Surgical technique: A circumcising incision was made and the penis was degloved. Buck's fascia was incised exposing the tunica albuginea opposite the curvature. 8 dots were marked bilaterally on the tunica albugineaand a $5 \mathrm{~mm}$ incision along the marks was made without cutting into the Corpus cavernosum. Instead of plicating with one suture for 4 dots, our modification uses one suture for two dots with the knot buried in a shallow trough created by a scalpel. Results: The average operation time was 64 minutes. We observed a loss of penile length in $30 \%$ of the patients $(0.5-1 \mathrm{~cm})$. There were no problems with erectile function. In a follow-up of 2 years, $90 \%$ of the patients remained without recurrence of deviation. None of patients reported problems with the suture knots. Conclusion: Our technique achieves penile straightening with minimal loss of length and no erectile dysfunction.
\end{abstract}

Keywords: Congenital Penile Deviation; Acquired Penile Deviation; Peyronie’s Disease; Penile Shortening

\section{Introduction}

Penile deviation (PD) is not only a cosmetic issue, but can also cause severe psychological problems in the affected patients. It can negatively affect the quality of the person's sexual life or even ruin a partnership [1,2]. Obviously, this disorder has both medical and social implications. There are two types of PD: congenital and acquired. Congenital PD's are anomalies of usually unknown aetiology. These patients present at young age with more than average penile length but perceive their PD as a psychologically detrimental condition. Congenital PD may be severe enough to preclude vaginal intercourse and thus compromise the normal sexual development of a young man [2, 3].

Acquired PD presents later in life. In most cases the curving of the penis is caused by Peyronie's disease. This form of penile deformation was first reported and described by Francois Gigot de la Peyronie in 1743 [4]. It is characterised by formation of fibrous plague on the cavernous body and can cause a permanent condition of PD.

There is no effective conservative treatment for congenital PD. Acquired PD has also been difficult to treat conservatively despite a plethora of oral and administered

${ }^{*}$ Corresponding author. therapies [5-8]. Most authorities believe acquired PD should be surgically corrected only if the deviation is severe enough to cause difficulty with intercourse.

There are many surgical techniques for PD correctionas well as different combinations of resection and plication procedures [8-15]. The earliest described correction was the Nesbit technique, which was originally suggested for congenital PD [16]. The Nesbit procedure consists of ellipsoid resection of the tunica albuginea opposite the curvature and suturing of the resected margins in order to compensate for a deficit of penile length caused by the plague [17]. A similar attack on the long side of the curvature with a plication technique is the Schroeder-Essed technique. This simple technique creates shallow incisions without resecting the tunica $[18,19]$.

In our practice plication techniques are used for all congenital and most acquired PD. However, the plication procedure can involve some well-known technical problems: the challenge is to avoid the development of bulges, which can cause bothersome sensations due to suture knots, and a loss of penile length. We therefore propose the "Kiel Knots" technique, which is based on a combination of the 16 dot plication procedure by T. Lue and the SchroederEssed technique [19,20]. 


\section{Materials and Patients}

Twenty patients with penile deviation, underwent the "Kiel Knots" plication technique, by the same surgeon. The average age 36.8 years (range 24 - 52 and follow-up was 26 months on average.In 8of the 20 patients the deviation was congenital, in 12 patients it was an acquired deviation. Patients with Peyronie's disease had stable disease condition for at least 6 months. The deviation ranged from 30 to 100 degrees, with an average of $72^{\circ}$. Patients with poor and moderate erectile function were excluded from the study. None of the selected patients presented with hourglass or hinge effect deformity. Photographs of the erect penis were taken in all patients documenting three different angles.

Congenital PD patients were not treated conservatively. Most acquired PD received oral therapy with Vitamin E in combination with Pentoxifyllin $400 \mathrm{mg}$ three times a day, for at least 6 months [21]. Surgery was only performed in patients with a consistent penile condition of at least 6 months. All patients had ultrasound examination of the penis for identification of plaque position and approximate size. Alprostadil was used to create an erection for photography and penile length measurement. Duplex Doppler was used to exclude any patients with moderate or severe erectile dysfunction (ED).

All patients and their partners were extensively informed about possible complications such as penile shortening, recurrent deviation, injury of the neurovascular bundle, infections, bleeding, and circumcision. Moreover we informed them that this operation technique is new and that we have no follow-up data on it. We also informed the patients about alternative classical ways of penile correction. As we mentioned, the described surgical technique is based on the two surgical techniques of T. Lue and Schroeder-Essed, which are standard procedures. But nevertheless, we have applied for additional approval of the university ethics committee for future procedures.

\section{Surgical Technique}

Surgery was performed under general anaesthesia in all cases although plication procedures are described in the literature under local and block anesthesia [22]. The procedure was performed under 3.5-fold optical magnification by means of operative spectacles (Teleflex, USA). A 16 French silicon transurethral catheter was placed in the bladder. None of the patients had previously been circumcised. Depending on the patients' wish, all patients underwent either full or partial circumcision with penile degloving of the skin. After circumcision, we placed a tourniquet around the penile base. Then 30 - $40 \mathrm{ml} \mathrm{NaCl}$ $0.9 \%$ solution was injected from the sulcus coronarius into the corpus cavernosum with a 18 gauge needle to enable detailed examination of the penile curvature under a simu- lated full erection. Alternatively, an artificial erection can be created by an injection of PGE 1 .

\subsection{Ventral Curvature}

Buck's fascia is dissected longitudinally from the sulcus coronaries to the base of the penis until tunica albuginea and neurovascular bundle are visualized. Then a bilateral intervascular space between the dorsal vein and the dorsal arteries is created as described by Lue and coworkers $[20,22]$. Alternately, a space for the dots is cleaned lateral to the neurovascular bundle. Then we use a surgical pen to mark 8 dots bilaterally (16 in total) beginning from the sulcus coronarius to the penile base. The distance between marked dots is approximately $5 \mathrm{~mm}$ (Figure 1) and 2 or 4 more dots may be required for the long penis.

A transverse incision of $5 \mathrm{~mm}$ is performed on the dots with a fine scalpel, without cutting into the corpus cavernosum (Figure 2). This is done to prepare a cavity for the inverted knots. Then the 000 double-needled GoreTex-suture is placed through the exterior edges of the two adjacent incisions. This differs from the classic 16 dots because a suture only plicates two dots instead of four (Figure 3). The double-armed needle is guided from outside to inside on either side so that the knot can be tied inside the incision (Figure 4). All sutures are knotted after placing of 4 pairs of sutureson each side (Figure 5). The inverted knot technique allows the surgeon to sink the knots into the shallow incision. In case parts of the suture remain on the surface, these can be pushed into the incision line with a forceps. Finally, the tourniquet is placed on the penis base again to assess the straightness of the penis after injection of $30-40 \mathrm{ml} \mathrm{NaCl} 0.9 \%$ into the corpus cavernosum. In case of over-correction, it is possible to undo one of the knots. In case of under-correction, 2 more dots and a further knot can be added.

\subsection{Dorsal Curvature}

Buck's fascia is dissected bilaterally, parallel to the urethra. exposing tunica albuginea. An incision of $5 \mathrm{~mm}$ is made on the dots with a fine scalpel without cutting into the Corpus cavernosum. The distance between the marked dots is again approximately $5 \mathrm{~mm}$ and inverted plication sutures are performed with 000 Gore-Tex-sutures. The distance between the knots and corpus spongiosum is approximately $3 \mathrm{~mm}$. 8 suture knots are done in total (Figure 6). The straightness of the penis is evaluated as described above. It is important to close Buck's fascia completely.

\section{Results}

The average operation time was 64 minutes. All patients underwent follow-up examinations at 6 monthly intervals. Minimum follow-up time was 26 months. 


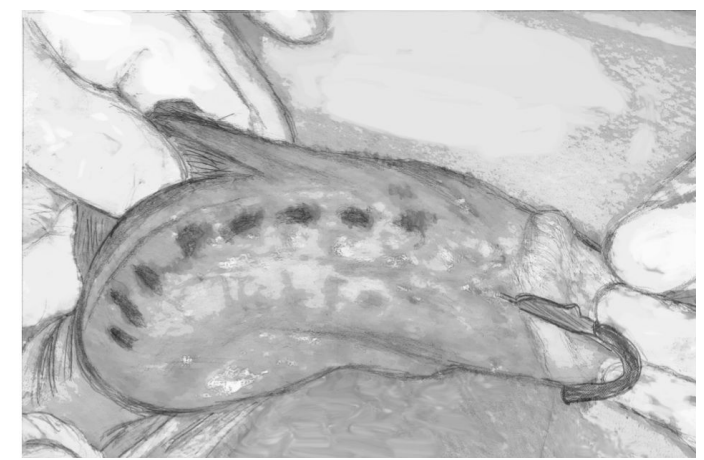

Figure 1.8 dots bilaterally (16 in total).

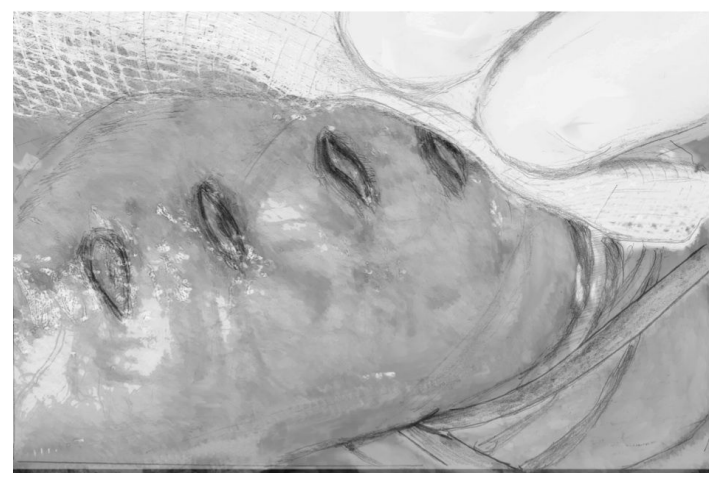

Figure 2. A transversal incision on the dots.

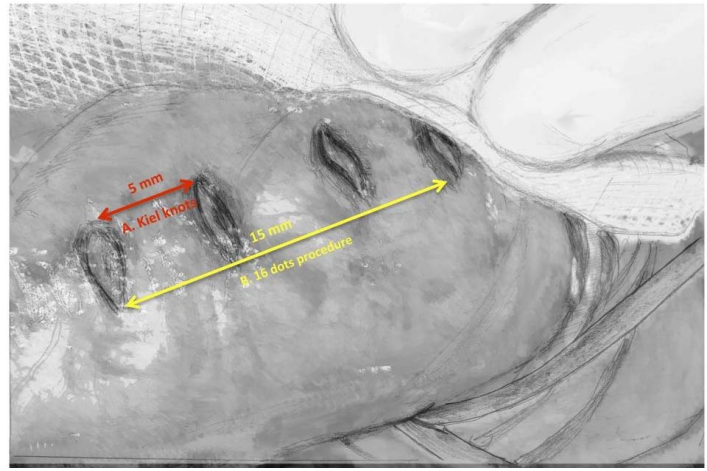

Figure 3. A distance between the dots (A) kiel knots procedure, (B) 16 dots procedure.

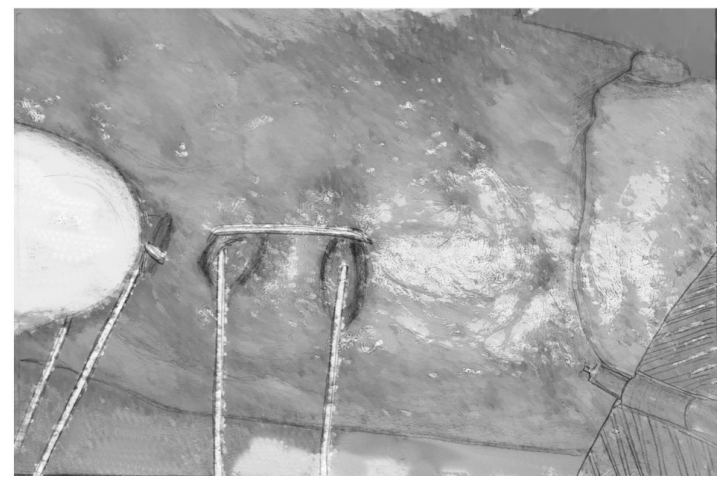

Figure 4. The $3 \times 0$ double needle Gore-Tex-suture $\mathrm{e}$ is guided form outside to inside.

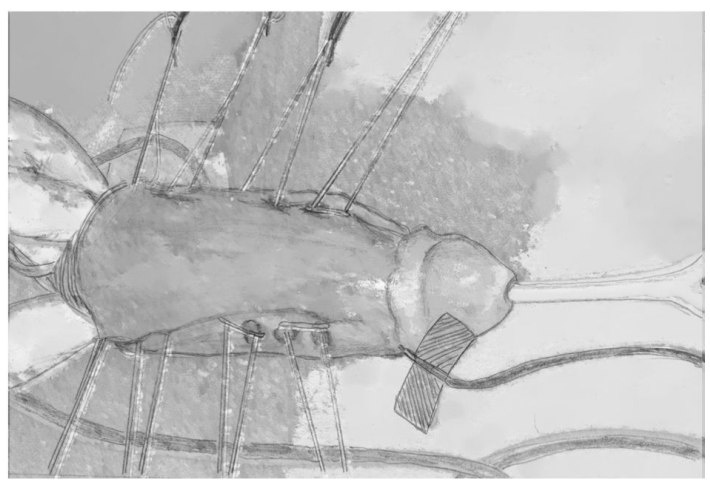

Figure 5. Ventral curvature.

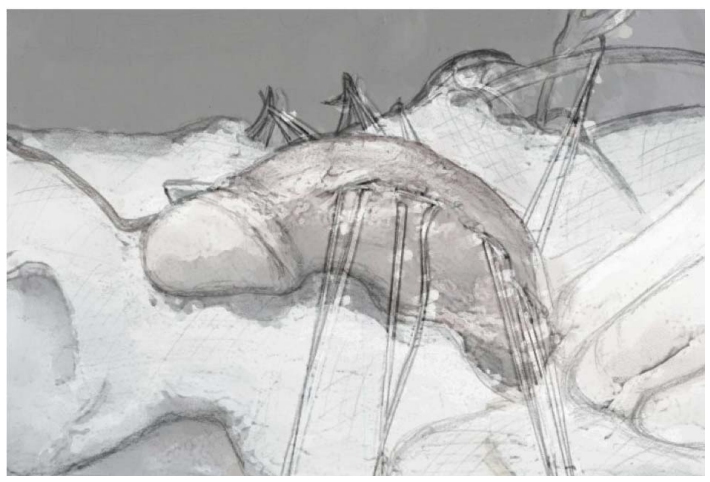

Figure 6. Dorsal curvature.

In the first 8 months none of the patients reported recurrence of PD. Two patients (10\%) reported a mild recurrence of PD after 24 months. Three patients (15\%) showed moderate ED one year postoperatively in an IIEF questionnaire but they were all acquired PD and had shown mild ED on the Doppler. A total of 18 patients (90\%) were satisfied with the cosmetic result. Penile shortening was the most frequent complication in the first 6 months, reported by 7 patients (35\%). All of these patients had had a moderate PD of less than $60^{\circ}$ [23]. One of these 7 patients found that sexual activity was affected by loss of penile length. After 6 months, however, penile shortening was no longer reported by any patient.

We attempted to objectify the penile shortening with measurement of the erect penis. After 26 months, penile shortening was observed in 6 patients (30\%). Loss of penile length ranged from 0.5 to $1 \mathrm{~cm}$.

This series was notable that none of the patients developed bulges seen with other plications and in no patients were the sutures palpable.

One of the 2 patients with mild PD recurrence underwent salvage surgery by Schroeder Essed technique. The other is under treatment with a vacuum constriction device. In the group of 6 patients with penile shortening, one patient underwent scrotoplasty, 5 patients are using a vacuum constriction device. The patients developing ED were treated with a regular intake of low dose Sildenafil. 
In one of them we could not find any correlation between the operation and his ED. Duplex Doppler showed no vascular problems and sufficient fuel ability of the corpora cavernosa. This patient also suffers from chronical depression and is under long-time psychological treatment. Therefore, we believe that his problems are more likely to be caused by psychological problems.

\section{Discussion}

Surgical correction of PD is a work in progress. Anytime there are so many operations described in the literature for one condition it is obvious consensus has not been achieved for correction of the deviation. In the long-term follow-up of our earlier modified Schroeder-Essed technique, noted a high satisfaction rate (93\%) of the patients [20]. However, we still faced problems in patients with extreme forms of PD, especially of congenital PD. The plication technique in those patients can often cause bulges, deformations and a relatively significant loss of penile length. We believe the problem is known: the longer the distance you have to plicate, the bigger the risk of bulge deformations. This could be effectively minimized by shortening the sections that are plicated or gathered.

For this reason we adapted the 16 dots procedure allowing consistent short gathers of tissue. The advantages of this technique are: 1) The points for incisions can easily be located; 2) It is ideal for treating advanced curvature as opposed to the Schroeder-Essed plication due to the equal distance between knots and the symmetrical distribution of placations; 3) Placement of the knots on the dorsal surface of the penis between the dorsal vein and the dorsal arteries or lateral to the bundle should prevent nerve injury.

When using the Schroeder-Essed plication, the position of the knots needs be approximated prior to suturing by using Allis clamps before finding the optimal position, especially in advanced curvature [19]. When using the 16dots procedure, this is not necessary, as the sutures are placed in consistent position with safely [22]. An added advantage is there are always 4 plications on the cavernous body and not one or two which prevents the development of the bulge phenomenon.

Another disadvantage of the Schroeder-Essed is the problem of palpable suture knots and discomfort during intercourse. This problem is potentially present for the 16dot but is probably not a factor since there are only two knots on each side of the penis. Inversion of the suture knots into the trough and substitution of $3 \times 0$ Gore Tex for Ticron seems to eliminate this real or perceived disadvantage.

We believe by using more sutures to plicate the 16 dots contributes to less shortening and parallel bulging as seen in Figure 3.

\section{Conclusion}

The "Kiel Knots" enhancement to a combination of the 16-dot and Schroder Essedplications has advantage of good straightening of penile curvature without excessive loss of penile length. It is advantageous because the bulges and palpable knots accompanying Schroder Essed are not seen and the penile shortening seemsminimal. We believe that our technique is primarily indicated in patients with extended penile deviations. Our experience so far leads us to suggest that it could be an effective technique in cases of congenital ventral and dorsal deviations. Additionally, we propose this method for patients with Peyronie's disease. Another advantage of our technique is that it is very simple and can be easily done with little or no complicatons. We are going to update our data as soon as we have performed a larger number of operations and with a longer follow up times. This being the case, we believe that we can further expand the scope of this technique.

\section{REFERENCES}

[1] R. Tal, O. Nabulsi, C. J. Nelson and J. P. Mulhall, “The Psychosocial Impact of Penile Reconstructive Surgery for Congenital Penile Deviation," The Journal of Sexual Medicine, Vol. 7, No. 1, 2010, pp. 121-128. doi:10.1111/j.1743-6109.2009.01574.X

[2] J. F. Smith, T. J. Walsh, S. L. Conti, P. Turek and T. Lue, "Risk Factors for Emotional and Relationship Problems in Peyronie's Disease,” The Journal of Sexual Medicine, Vol. 5, No. 9, 2008, pp. 2179-2184. doi:10.1111/j.1743-6109.2008.00949.x

[3] O. Shaeer and K. Shaeer, "The Global Online Sexuality Survey (GOSS): Erectile Dysfunction among ArabicSpeaking Internet Users in the Middle East,” The Journal of Sexual Medicine, Vol. 8, No. 8, 2011, pp. 2152-2160. doi:10.1111/j.1743-6109.2011.02297.x

[4] François Gigot de la Peyronie, "Sur Quelques Obstacles, qui S’Opposent à L’Ejaculation Naturelle de la Semence,” Mémoires de l’Académie Royale de Médecine, Paris, 1743, pp. 425-434.

[5] R. E. Fricke and J. H. Varney, "Peyronie's Disease and Its Treatment with Radium,” Journal of Urology, Vol. 59, No. 4, 1948, pp. 627-630.

[6] V. Meineke, C. Uebler, F. M. Köhn, et al., "Radiotherapy in Benigndiseases: Morbus Peyronie,” Strahlentherapie und Onkologie, Vol. 179, No. 3, 2003, pp. 181-186.

[7] R. Dickstein, J. Uberoi and R. Munarriz, "Severe, Disabling, and/or Chronic Penile Pain Associated with Peyronie Disease: Management with Subcutaneous Steroid Injection,” Journal of Andrology, Vol. 31, No. 5, 2010, pp. 445-449. doi:10.2164/jandrol.109.008029

[8] R. T. Strebel, S. Suter, T. Sautter and D. Hauri, "Extra Corporeal Shock Wave Therapy for Peyronie's Disease Does Not Correct Penile Deformity," International Journal of Impotence Research, Vol. 16, No. 5, 2004, p. 4. 
[9] A. Müller and J. P. Mulhall, "Peyronie’s Disease Intervention Trials: Methodological Challenges and Issues," The Journal of Sexual Medicine, Vol. 6, No. 3, 2009, pp. 848-861. doi:10.1111/j.1743-6109.2008.01081.x

[10] L. A. Levine, "Partial Plaque Excision and Grafting (PEG) for Peyronie’s Disease,” The Journal of Sexual Medicine, Vol. 8, No. 7, 2011, pp. 1842-1845. doi:10.1111/j.1743-6109.2011.02370.x

[11] S. V. Perovic and R. P. Djinovic, "Current Surgical Management of Severe Peyronie's Disease,” Archivos Españoles de Urología, Vol. 63, No. 9, 2010, pp. 755-770.

[12] E. Chung, E. Clendinning, L. Lessard and G. Brock, "Five-Year Follow-Up of Peyronie’s Graft Surgery: Outcomes and Patient Satisfaction," The Journal of Sexual Medicine, Vol. 8, No. 2, 2011, pp. 594-600. doi:10.1111/j.1743-6109.2010.02102.x

[13] O. Shaeer, “Trans-Corporal Incision of Peyronie's Plaques," The Journal of Sexual Medicine, Vol. 8, No. 2, 2011, pp. 589-593. doi:10.1111/j.1743-6109.2010.02078.x

[14] M. L. Eisenberg, J. F. Smith, A. W. Shindel and T. F. Lue, "Tunica-Sparing Ossified Peyronie's Plaque Excision," BJU International, Vol. 107, No. 4, 2011, pp. 622-625. doi:10.1111/j.1464-410X.2010.09546.X

[15] G. Colpi, G. Piediferro, F. Castiglioni, G. Contalbi and L. Carmignani, "Penile Septoplasty for Congenital Ventral Penile Curvature: Results in 51 Patients," Journal of Urology, Vol. 182, No. 4, 2009, pp. 1489-1494. doi:10.1016/j.juro.2009.06.059

[16] R. H. Nesbit, "Congenital Curvature of the Phallus: Report of Three Cases with Description of Corrective Ope- ration,” Journal of Urology, Vol. 93, 1965, pp. 230-232.

[17] R. J. Lemberger, M. C. Bishop and C. P. Bates, "Nesbit's Operation for Peyronie's Disease," British Journal of Urology, Vol. 56, 1984, pp. 721-723. doi:10.1111/j.1464-410X.1984.tb06156.x

[18] E. Essed and F. H. Schröder, "New Surgical Treatment for Peyronie's Disease,” Urology, Vol. 25, No. 6, 1985, pp. 582-587. doi:10.1016/0090-4295(85)90285-7

[19] C. van der Horst, F. J. Martinez Portillo, C. Seif, et al., "Tunica Plication with Horizontal Incisions of the Tunica Albuginea in the Treatment of Congenital Penile Deviations,” Aktuelle Urologie, Vol. 34, No. 7, 2003, pp. 478480.

[20] L. S. Baskin and T. F. Lue, "The Correction of Congenital Penile Curvature in Young Men,” British Journal of Urology, Vol. 81, No. 6, 1998, pp. 895-899. doi:10.1046/j.1464-410x.1998.00645.X

[21] J. F. Smith, A. W. Shindel, Y. C. Huang, et al., "Pentoxifylline Treatment and Penile Calcifications in Men with Peyronie's Disease,” Asian Journal of Andrology, Vol. 13, No. 2, 2011, pp. 322-325. doi:10.1038/aja.2010.117

[22] S. S. Gholami and T. F. Lue, "Correction of Penilecurvature Using the 16-Dot Plication Technique: A Review of 132 Patients,” Journal of Urology, Vol. 167, No. 5, 2002, pp. 2066-2069. doi:10.1016/S0022-5347(05)65085-9

[23] T. Akman, L. Gurkan, O. Sanli, K. Onem, M. Tezer and A. Kadioglu, "Clinical Characteristics of Pure Notching Group in Peyronie's Disease,” Program and Abstracts of the Sexual Medicine Society of North America Fall Meeting, New York, 17-20 November 2005. 